\title{
Welche Subgruppe profitiert?
}

Fragestellung: Ist es möglich Subgruppen von Patienten mit Glioblastom zu identifizieren, die von einer kombiniert antiangionetisch/alkylierenden Chemotherapie mit Bevacizumab und Lomustin (CCNU) profitieren?

Hintergrund: Antiangionetische Therapien wie die Inhibition des Vascular Endothelial Growth Factor (VEGF) durch den humanisierten Antikörper Bevacizumab sollten aufgrund der auch histologisch die Diagnose eines Glioblastoms definierenden Neovaskularisation ein potentes therapeutisches Ziel darstellen. Bisherige Studien in der Primärtherapie waren jedoch enttäuschend und konnten insbesondere keinen Vorteil für die Über-

lebenszeit dokumentieren. In der niederländischen BELOB-

Erdem-Eraslan L, van den Bent MJ, Hoogstrate $Y$ et al. Identification of patients with recurrent glioblastoma who may benefit from combined bevacizumab and CCNU therapy: A report from the BELOB trial. Cancer Res 2016; DOI: 10.1158/0008-5472.CAN-15-0776 Studie gelang das zum ersten Mal bei Patienten mit rezidiviertem Glioblastom mit einer Kombination aus Bevacizumab und CCNU [1].

Patienten und Methodik: Es waren 114 von ursprünglich 152 Tumorblöcken, der an der
BELOB-Studie teilnehmenden Patienten zur molekulargenetischen Untersuchung verfügbar. RNA-Expressionsprofile wurden einem von sechs Intrinsic Glioma Subtypes (IGS-0, -9, -17, -18, $-22,-23)$ oder einer von vier durch „The Cancer Genome Atlas“ (TCGA) definierten molekularen Subgruppen (proneural, neural, klassisch und mesenchymal) zugeordnet. Die statistische Bedeutung unterschiedlicher prognostischer Faktoren wurde mit der Cox-Regression untersucht und die Signifikanz von unterschiedlichen Überlebenszeiten in den Kaplan-Meier-Kurven durch den Log-Rank-Test bestimmt.

Ergebnisse: Patienten mit Glioblastomen, die dem IGS-18 oder dem klassischen Subtyp zugeordnet werden konnten, zeigten ein signifikant verlängertes progressionsfreies Überleben und einen Trend zu einem Vorteil im Gesamtüberleben unter der kombinierten Rezidivtherapie mit Bevacizumab und CCNU. Die Expressionen von FMO4 und OSBPL3 waren ebenfalls mit einem Ansprechen auf die Kombinationstherapie assoziiert.

Schlussfolgerungen: Sollten sich die Ergebnisse in einer unabhängigen Kohorte bestätigen lassen, wäre es möglich, Glioblastome hinsichtlich ihres Ansprechens auf eine Kombinationstherapie mit Bevacizumab und CCNU zu selektionieren.

\section{- Kommentar von Martin Uhl, Erlangen}

\section{Die Zukunft von Bevacizumab erscheint ungewisser denn je}

Der Versuch, negativen Studienergebnissen mit einer Subgruppenanalyse noch Positives abzugewinnen, hat eine lange Tradition. Bereits bei den negativen Bevacizumab-Studien Avaglio und RTOG 0825 wurde in der Primärtherapie versucht, eine molekulare Subgruppe zu definieren, die ein Überlebensvorteil vorhersagen soll. Bei Avaglio war das der „proneurale“ Subtyp, der besonders gut ansprach [2], wohingegen bei RTOG 0825 der „mesenchymale“ Subtyp [3] besonders schlecht war. Die Arbeit von Erdem-Eraslan et al. ist ungewöhnlich, da sie ja eine positive Studie zugrunde liegen hat, dann einer Subgruppenanalyse unterzogen wird und nur noch einen Trend $z u$ einem verlängerten Überleben zeigt? Die Rationale hierfür erschließt sich erst, wenn man weiß, dass die zugehörigen Phase-III-Studien der EORTC 26101 die BELOB-Daten kürzlich nicht bestätigen konnten [4]. Es wurden nun neben dem „klassischen" Subtyp auch die zwei Gene FMO4 und OSBPL3 gefunden. Diese sind mit dem Metabolismus von Medikamenten, der Regulation des Aktinzytoskeletts und der Zelladhäsion assoziiert und können den biologischen Ansatz einer Kombination aus Antiangionese und alkylierender Chemotherapie gut erklären. Der "klassische“ Subtyp muss auch nicht in offenem Widerspruch zu den oben genannten Daten stehen. Da es sich um eine Studie bei rezidivierten Glioblastomen handelt, wäre hier eine negative Selektion der prognostisch schlechten Subgruppen zu diskutieren. Inwieweit sich diese Ergebnisse nun zeit- nah auf den klinischen Alltag auswirken ist fraglich. Eine Bestimmung der genannten Subgruppen erfolgt momentan nur als wissenschaftliches Begleitprogramm und kann auf absehbare Zeit wohl nicht zur Therapieentscheidung herangezogen werden. Insgesamt erscheint mir die Zukunft von Bevacizumab ungewisser denn je, da auch die GLARIUS-Studie [5], die sich auf die prognostisch ungünstige Subgruppe der nicht MGMTPromoter-methylierten Gliblastome fokussierte, negativ war.
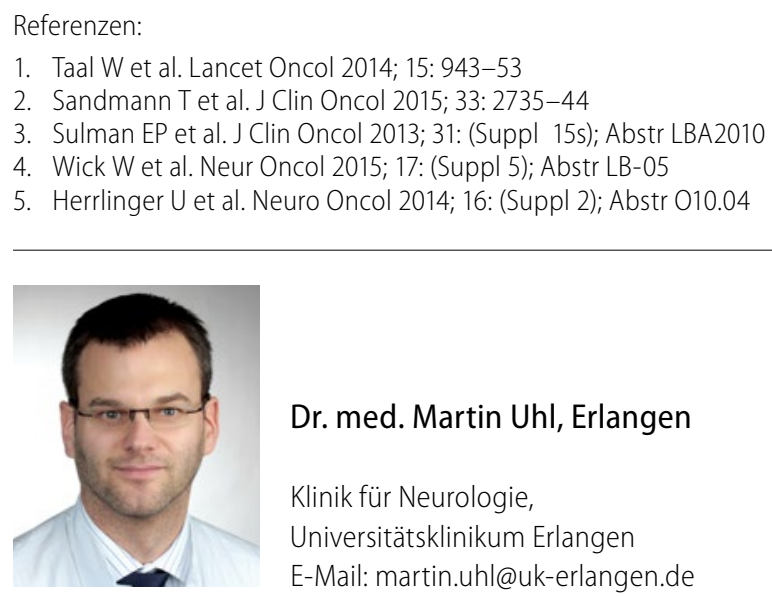

Dr. med. Martin Uhl, Erlangen

Klinik für Neurologie, Universitätsklinikum Erlangen E-Mail:martin.uhl@uk-erlangen.de 